\title{
A Comparative Study Among Different Invasive Methods For The Diagnosis Of Helicobacter Pylori
}

\author{
MDU Islam ${ }^{1}$, SHZ Rahman², SM Shamsuzzaman ${ }^{3}$, N Muazzam4, SG Kibria ${ }^{5}$, MT Hossain ${ }^{6}$, N Ahmed ${ }^{7}$, ARSarkar ${ }^{8}$, S Nahar $^{9}$
}

\begin{abstract}
H. pylori infection occurs worldwide. Approximately $50 \%$ of the world population is infected with this organism. A cross-sectional study was conducted on 81 dyspeptic patients attending at Dhaka Medical College Hospital, for diagnostic endoscopy. H. pylori infection was measured by three invasive methods: culture, rapid urease test \& histopathology. Among study patients maximum $(34.57 \%) \mathrm{H}$. pylori infected were in 21-30 years age group, bearing male female ratio 1: 92.86 . Forty four (54.32\%) out of 81 were culture positive, $61(75.31 \%)$ were rapid urease test positive and 62 (76.54\%) were histopathology positive. By using 'gold standard' definition, 64 (79.02\%) were H. pylori infected, 17 were un-infected. Finally comparing among three invasive methods, all are highly sensitive and specific to diagnose $\mathrm{H}$. pylori infection.
\end{abstract}

\section{Introduction}

In 1983 an Australian pathologist, Dr J Warren, reported an association between colonization of the stomach with curved bacilli, currently known as Helicobacter pylori and the presence of active gastritis ${ }^{1}$. Significant correlation between $\mathrm{H}$. pylori-associated gastritis and peptic ulcer has been found ${ }^{2}$. Gastric cancer and lymphoproliferative gastric diseases also have been correlated with H. pylori infection ${ }^{3}$.Consequently, many histopathologists and microbiologist are being asked to detect infection with this organism, but there is no commonly acknowledged "Gold standard" method for diagnosing H. pylori infection.

1. Dr. Md. Din-U1 Islam, M Phil (Microbiology), Lecturer, Dept. of Microbiology, FMC, Faridpur.

2. Dr. Sufi Hannan Zulfiqar Rahman, MD (Immunology), Assistant Professor, Dept. of Immunology \& Molecular Biology, NICR\&H, Mohakhali, Dhaka.

3. Dr. Sheikh Mohammad Shamsuzzaman, M Phil (Microbiology), $\mathrm{PhD}$, Associate Professor, Dept. of Microbiology, DMC, Dhaka.

4. Dr. Naima Muzzam, M Phil (Microbiology), Professor, Dept. of Microbiology and Mycology, NIPSOM, Mohakhali, Dhaka.

5. Dr. Syed Golam Kibria, M Phil (Pathology), Professor \& Head, Dept. of Pathology, FMC, Faridpur.

6. Dr. Md. Tofazzal Hossain, MD (Pathology), Lecturer, Dept. of Pathology, FMC, Faridpur.

7. Dr. Nasim Ahmed, M Phil (Pathology), Associate Professor, Dept. of Pathology, Delta Medical College, Dhaka.

8. Dr. Abdur Rab Sarkar, MCPS (Medicine), MD (Gastro), Associate Professor, Dept. of Gasstroenterology, DMC, Dhaka.

9. Mrs. Shamsun Nahar, M Sc, Associate Scientist, Laboratory Science Division of ICDDR,B, Mohakhali, Dhaka

\section{Address of Correspondence}

Dr. Din-U1 Islam, Lecturer, Dept. of Microbiology,

Faridpur Medical College, Faridpur.

Mobile: +88-01552420598, E-mail: dr.dinul.islam@gmail.com.
There are several diagnostic tools, which include invasive and non-invasive methods, for the diagnosis of $\mathrm{H}$. pylori infection, are available. But all of the tests have their pitfalls and limitations. Invasive tests, such as culture, histopathology and biopsy urease test require endoscopic biopsy of gastric tissue. Culture allows testing for susceptibility of antimicrobials and its sensitivity \& specificity is $77-95 \%$ \& $100 \%$ respectively. Rapid urease test is a qualitative assay for the detection of urease and its sensitivity \& specificity are $89-98 \%$ \& $93-98 \%$ respectively. Histopathology by haematoxylin-eosin \& modified Giemsa staining is important to detect $\mathrm{H}$. pylori under light microscope \& its sensitivity \& specificity are $93-98 \%$ \& $95-98 \%$ respectively ${ }^{4}$.

In a study at BIRDEM, the positivity of culture is $59.3 \%$, for rapid urease test it is $60.4 \%$, for histopathology it is $34.4 \%{ }^{5}$. Till to date, invasive tests have been considered as the gold standard tests but these biopsy based methods may suffer from sampling errors, because of the patchy nature of the infection and low concentration of bacteria in the fragments ${ }^{6}$. Culturing also has a low sensitivity, therefore a combination of the tests is recommended as gold standard ${ }^{7}$.

There are several non-invasive methods including urea breath test, H. pylori Ag detection in stool, H. pylori IgG $\mathrm{Ab}$ detection in urine, serum and saliva, are available for the diagnosis of, H. pylori infection. Each has its own advantage, disadvantage and limitations ${ }^{8}$. So this study has been designed and carried out to compare the results of culture, rapid urease test and histology in diagnosing $\mathrm{H}$. pylori infection. 


\section{Materials and methods}

This cross sectional type of study was carried out in the Department of Microbiology of Dhaka Medical College, in collaboration with Dept. of Gastroenterology of Dhaka Medical College Hospital and Laboratory Sciences Division of ICDDR,B from January 2007 to December 2007. A total of 81 adult patients with dyspeptic symptoms, referred for upper gastrointestial endoscopy were studied. Patients excluded from the study were (i) Patients who had partial or complete gastrectomy or gastro-jejunostomy (ii) Patients who had ever received $H$. pylori eradication therapy (iii) Patients who had taken any antibiotic, colloidal bismuth compound, proton pump inhibitor in last one month (iv) Patients with bleeding peptic ulcer (v ) Chronic user of corticosteroids or immunosuppressive drugs ${ }^{9}$.

\section{Sample collection}

Gastric biopsy: From each selected patient three gastric biopsies were taken with biopsy forceps. Two biopsies, one from antrum and another from corpus, were taken for both culture and for rapid urease test and were put in two separate microcentrifuge tubes. These samples were then transported to the $\mathrm{H}$. pylori laboratory of ICDDR,B in a cool box. Another biopsy from antrum was taken for $\mathrm{H} \& \mathrm{E}$ and Giemsa stain and was preserved in a separate microcentrifuge tube containing $10 \%$ formalin.

Culture: In the laboratory, two microcentrifuge tubes containing gastric biopsy specimens, were vortexed vigorously for 5 minutes. From each microcentrifuge tube $200 \mu \mathrm{l}$ of brain heart infusion (BHI) broth were taken and plated on brain heart infusion agar plates containing $7 \%$ sheep blood, $0.4 \%$ IsovitaleX and $\mathrm{H}$. pylori selective (Dent) supplement. The plates were incubated at $37^{\circ} \mathrm{C}$ in a $\mathrm{CO}_{2}$ water jacketed incubator for 3-7 days. Positive cultures were identified by-Colony morphology, Gram stain morphology, Positive catalase test, Positive oxidase test, Strong urease activity.

Biopsy urease test: From two separate transport media containing gastric biopsy specimens, $100 \mu \mathrm{BHI}$ broth were taken and inoculated in to Christensen's urea broth media in microcentrifuge tubes separately. Tubes were kept at room temperature. Change of colour from yellow to pink by any specimen within 24 hours was considered as positive rapid urease test.

Histopathology: One gastric biopsy specimen from the antrum was stained H\&E and modified Giemsa. Stained slide were examined under light microscope to find out curved spiral shaped H. pylori bacilli.

Definition of $\mathrm{H}$. pylori infection was 'Patients with positive culture result were considered as infected. In case of negative culture, patients positive by both biopsy urease test and histology were considered as infected. Patients negative by all three gastric biopsy based tests i.e. culture, urease test and histology were considered as uninfected. Patients negative by culture and positive by either biopsy urease test or histology were considered as indeterminate. ${ }^{10}$.

\section{Results}

Total 81 patients with dyspeptic symptoms were selected for the study. Table I shows the age distribution of the selected patients. They were aged between 16 to 70 years with a mean age of 37.98 years.

Table I: Age distribution of the study population $(n=81)$

\begin{tabular}{lcc}
\hline Age group(years) & No of patients & Percentage \\
$\leq 20$ & 5 & 6.17 \\
$21-30$ & 28 & 34.57 \\
$31-40$ & 20 & 24.69 \\
$41-50$ & 11 & 13.58 \\
$51-60$ & 11 & 13.58 \\
$61-70$ & 6 & 7.41 \\
\hline Total & 81 & 100.00 \\
\hline
\end{tabular}

Table II shows the sex distribution of the study patients. Of the 81 patients, $42(51.85 \%)$ were males and 39 $(48.15 \%)$ were females with a male female ratio 1 : 92.86.

Table II: Sex distribution of the study population $(\mathrm{n}=81)$

\begin{tabular}{llc}
\hline Sex & No of patients & Percentage \\
\hline Male & 42 & 51.85 \\
Female & 39 & 48.15 \\
\hline Total & 61 & 100.00 \\
\hline
\end{tabular}

Fig. 1 shows H. Pylori positivity by different tests. Bar diagram shows percentage of patients positive for $\mathrm{H}$. pylori, in different methods. Of the 81 patients, 44 $(54.32 \%)$ were culture positive, 61 (75.31\%) were rapid urease test positive, $62(76.54 \%)$ were histopathological stained positive for $\mathrm{H}$. pylori. 


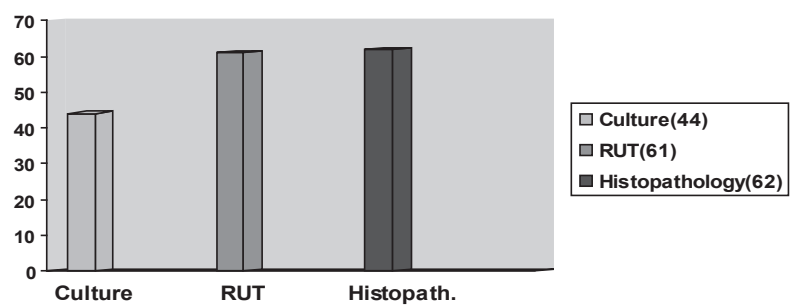

Figure1: Bar chart showing culture, RUT and Histopathological stained positive H. pylori infection of biopsy tissue

Table III Shows the comparison of culture, rapid urease test and histopathology for the detection of H. pylori infection. Among the 44 culture positive cases, 37 were RUT \& histopathologically positive for $\mathrm{H}$. pylori infection. Among 37 culture negative cases, 20 were RUT \& histopathologically positive. Total number of infected cases were 64 . Fourteen were uninfected and 3 were indeterminate, one of them was only RUT positive and 2 were only histopathologically positive.

Table III: Comparison of culture, rapid urease test and histopathology for the detection of $\mathrm{H}$. pylori infection

\begin{tabular}{lccccc}
\hline $\begin{array}{l}\text { Culture } \\
\text { results }\end{array}$ & $\begin{array}{c}\text { RUT\&Histopath } \\
\text { Positive }\end{array}$ & $\begin{array}{c}\text { RUT\&Histopath } \\
\text { Negative }\end{array}$ & $\begin{array}{c}\text { RUT } \\
\text { Positive }\end{array}$ & $\begin{array}{c}\text { Histopath } \\
\text { Positive }\end{array}$ & $\begin{array}{l}\text { H. pylori } \\
\text { infection }\end{array}$ \\
\hline $\begin{array}{l}\text { Culture } \\
\text { positive } \\
(\mathrm{n}=44)\end{array}$ & 37 & 1 & 3 & 3 & 44 \\
$\begin{array}{l}\text { Culture } \\
\text { negative } \\
(\mathrm{n}=37)\end{array}$ & 20 & 14 & 1 & 2 & 20 \\
\hline Total & 57 & 15 & & & \\
\hline
\end{tabular}

Table IV shows $\mathrm{H}$. pylori infection among study patients. Among 81 patients, 64 (79.02\%) were infected, 14 (17.28\%) were uninfected and $3(3.70 \%)$ were indeterminate.

Table IV: H. pylori infection state of the study population $(\mathrm{n}=81)$.

\begin{tabular}{lcc}
\hline H. pylori infection & No of patients & Percentage \\
\hline Infected & 64 & 79.02 \\
Uninfected & 14 & 17.28 \\
Indeterminate & 3 & 3.70 \\
\hline Total & 86 & 100.00 \\
\hline
\end{tabular}

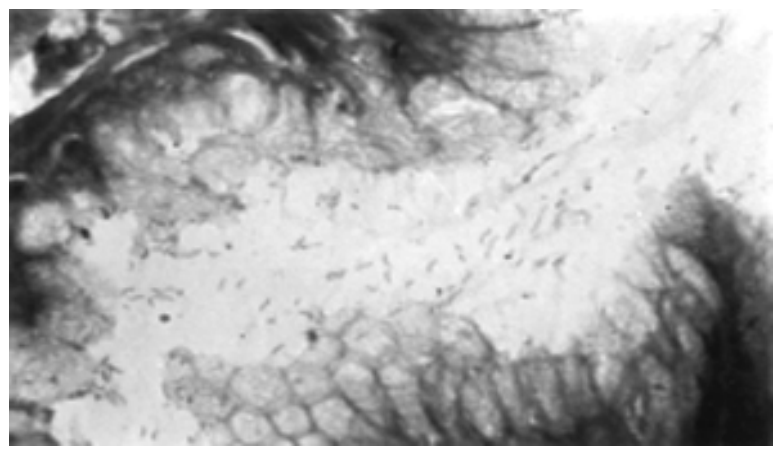

Figure 2: Photomicrograph of histopathological section of gastric mucosa showing plenty of $\mathrm{H}$. pylori over the surface. Stain: Modified Giemsa stain, Magnification: $x$ 1000 (Picture in Black \& White)

\section{Discussion}

Helicobacter pylori live on gastric mucosa, especially on gastric antrum in between the epithelial surface and mucus overlying the mucosa where $\mathrm{p}^{\mathrm{H}}$ is about seven ${ }^{11}$.

In this study, out of 81 dyspeptic cases, highest incidence $(34.57 \%)$ of $\mathrm{H}$. pylori infection was observed in 21-30 years age group (Table I). Literature study showed that sero-prevalence of $\mathrm{H}$. pylori is different among different age groups ${ }^{12}$. In USA, highest incidence $(24.5 \%)$ was reported in $40-50$ years age groups and lowest (03\%) in 10-20 years age group ${ }^{13}$. In Bangladesh, study showed maximum prevalence in 31$40(29 \%)$ year's age group ${ }^{14}$. In the present study, male $(51.85 \%)$ and female $(48.15 \%)$ ratio was 1: 92.86 (Table II). Alemohammad, et al. reported that, in a study in USA, $53.3 \%$ male and $46.7 \%$ female were $\mathrm{H}$. pylori infected ${ }^{13}$.

At present, many tests are available for diagnosis of $\mathrm{H}$. pylori infection. Invasive tests, such as culture, histopathology and biopsy urease test require endoscopic biopsy of gastric tissue. And non-invasive tests, such as urea breath test, stool antigen detection, antibody detection in saliva, in serum and in urine are available. Present study was aimed to compare the results of mostly used three invasive methods: culture, urea breath test and histopathological stained based detection of $\mathrm{H}$. pylori infection.

A total of $44(54.32 \%)$ out of 81 patients were positive by culture in this study (Fig.1). Similarly, in a study in Bangladesh, Rahman reported $59.03 \%$ positivity in culture ${ }^{5}$. Alemohammad, et al. reported $60 \%$ culture positivity among the people attending for upper GIT endoscopy in USA ${ }^{13}$. In Taiwan, Kuo, et al. 
reported $55.6 \%$ of culture positive cases among dyspeptic patients ${ }^{9}$. The less number of culture positivity might be due to the fact that distribution of $\mathrm{H}$. pylori in stomach may be patchy, a few sq. mm biopsy tissue from 800 sq. cm mucosal surface of stomach may not contain $\mathrm{it}^{14}$.

In this study, 61 (75.31\%) out of 81 patients were rapid urease test positive (Fig.1). In a study in USA, rapid urease test was positive in $67 \%$ of the dyspeptic patients $^{13}$. In Bangladesh reported $60.4 \%$ rapid urease test positivity among dyspeptic patients 5 . These findings are consistent with the findings of the present study. In contrast to the findings of the present study, Sahidullah reported higher rapid urease test positivity $(91.17 \%)$ among fish handlers of Bangladesh ${ }^{15}$. This difference might be due to the fact that $\mathrm{H}$. pylori infection is more among fish handlers and biopsy materials were taken only from seropositive cases in that study.

In this study, $62(76.54 \%)$ of the 81 samples were histopathologically positive for $\mathrm{H}$. pylori. In a study in USA, H. pylorus was identified in stained biopsy specimen among $75 \%$ of the patients attending for endoscopy ${ }^{13}$. In contrast to the findings of the present study, Monterio, et al. reported $95.6 \%$ positivity for $\mathrm{H}$. pylori from stained gastric biopsy specimens among untreated patients in a study in France, which was higher than that of the present study ${ }^{16}$.

In this study, according to the standard definition ${ }^{10,16}$, all the 44 culture positive patients were $\mathrm{H}$. pylori infected. Among 37 culture negative cases, 20 were rapid urease test and histopathologically positive and were considered $\mathrm{H}$. pylori infected (Table III). As a result, among 81 dyspeptic study populations, 64 (79.02\%) were infected with $\mathrm{H}$. pylori (Table IV). Rahman reported that $68.1 \%$ of the dyspeptic patients in Bangladesh ${ }^{5}$ and Kuo, et al. reported that $72.6 \%$ of the dyspeptic patients in Taiwan were infected by $\mathrm{H}$. pylori using same definition ${ }^{9}$. In other study in USA, Alemohammad, et al. reported $77.12 \% \mathrm{H}$. pylori infection among patients attending endoscopy. These results coincide with the results of the present study ${ }^{13}$.

In summery, our results of invasive methods are highly sensitive and specific. The sensitivity and specificity of culture, rapid urease test and histopathological staining are $68.75 \% \& 100.00 \%, 93.75 \% \& 92.86 \%$ and $93.75 \%$ \& $85.71 \%$ respectively. Though these tests have sampling error and no single test is considered as gold standard, any of these invasive methods can be used for diagnosis of $\mathrm{H}$. pylori infection confidently for its high sensitivity and specificity.

\section{References}

1. Warren RJ, Marshall B. Unidentified curved bacilli on gastric epithelium in active chronic gastritis. The Lancet 1983;321(8336):1273-75.

2. Rautelin H, Kosunen TU. Helicobacter pylori and associated gastroduodenal diseases. APMIS 1991;99:677-695.

3. Wotherspoon AC, Ortiz- Hidalgo C, Falzon MR, Isaacson PG. Helicobacter pylori associated gastritis and primary B-cell gastric lymphoma. The Lancet 1991;338:1175-76.

4. Dunn BE, Cohen H, Blaser MJ. Helicobacter pylori. Clinical Microbiology Reviews 1997; 10(4):720-41.

5. Rahman SHZ. Immunoglobulin $\mathrm{G}$ (IgG) antibody response to Helicobacter pylori antigens and its correlation with gastric pathology. (MD thesis). Dhaka: Department of Immunology, BIRDEM; 2005.

6. Morris A, Ali MR, Brown P. Campylobacter pylori infection in biopsy specimen of gastric antrum: laboratory diagnosis and estimation of sampling error. J Clin Pathol. 1989;42:727-732.

7. Culter AF, Haustad S, Ma CK. Accuracy of invasive and noninvasive test to diagnose Helicobacter pylori infection. Gastroenterol. 1995;109:136-141.

8. Crabtree JE, Phil D, Shallcross TM. Mucosal human immune response to Helicobacter pylori in patients with dudenitis. Diges Dis \& Sci. 1991;36:1266-1273.

9. Kuo FC, Wang SW, Wu IC, Yu FJ, Yang YC, Wu JY, et al. Evaluation of urine test for detecting Helicobacter pylori infection in Taiwan: A prospective study. World Journal of Gastroenterology 2005;11(35):5545-48.

10. Frenck RW, Fathy HM, Sherif M, Mohran Z, Mohammedy HE, Francis W, et al. Sensitivity and specificity of various tests for the Diagnosis of Helicobacter pylori in Egyptian Children. American Academy of Pediatrics 2006;118(4):1195-02.

11. Skirrow MB. Campylobacter and Helicobacter. In: Greenwood D, Slack RCB, Peutherer JF (eds). Medical Microbiology, $15^{\text {th }}$ edition. Churchill Livingstone, London; 1997: pp. 290-297.

12. Ahmad MM, Rahman M, Rumi AK, Islam S, Huq F, Chowdhury MF, et al. Prevalence of Helicobacter pylori in asymptomatic population- a pilot serological study in Bangladesh. J Epidemiol. 1997;7(4):251-4.

13. Alemohammad MM, Foley TJ, Cohen H. Detection of Immunoglobulin G Antibodies to Helicobacter pylori in Urine by an Enzyme Immunoassay Method. Journal of Clinical Microbiology 1993;31(8):2174-77.

14. Ahmed N. Gastric metaplasia in the duodenum: Role of inflammation and Helicobacter pylori. (M.Phil thesis).Dhaka: Department of Pathology, BSMMU;1999.

15. Sahidullah S. Seropositivity of Helicobacter pylori among the fish handlers. (M.Phil thesis). Dhaka: Department of Microbiology, SSMC; 2007.

16. Monterio L, de Mascarel A, Sarrasqueta AM, Bergey B, Barbaris C, Talby P, et al. Diagnosis of Helicobacter pylori: Non-invasive methods compared to invasive and evaluation of two new tests. The American Journal of Gastroenterology 2001;96(2):353-58. 